\title{
ORIGINAL
}

\section{TUBERCULOSIS EN BARCELONA: MODELO PREDICTIVO BASADO EN SERIES TEMPORALES}

\author{
M. Pilar Muñoz $(1,2)$, Angels Orcau $(2,3)$ y Joan Caylà $(2,3)$ \\ (1) Departamento de Estadística e Investigación Operativa, Universitat Politècnica de Catalunya. \\ (2) CIBERESP. \\ (3) Servei d'Epidemiologia. Agència de Salut Pública de Barcelona.
}

\section{RESUMEN}

La tuberculosis (TB) a escala mundial sigue ocasionando cada año millones de casos nuevos y de muertes. La Organización Mundial de la Salud (OMS) ha estimado recientemente que en el 2007 se registraron 9.273.000 casos (incidencia de $139 / 100.000$ habitantes) y 1.772 .000 muertes atribuibles a esta vieja enfermedad, y en el año precedente la cifra absoluta fue ligeramente inferior $(9.240 .000$ casos $)$ mientras que la incidencia fue algo superior (140/100.000), por lo que se hace difícil precisar si hay declive o no. El objetivo de este trabajo es aplicar modelos predictivos a la TB, diferenciando entre población autóctona e inmigrante, en una ciudad en la que ha sido posible determinar el número anual de casos desde 1987.

Se ha ajustado una tendencia segmentada también denominada regresión discontinua a tramos (piecewise regression $\mathrm{o}$ segmented regression) a las series de casos nuevos en la población autóctona e inmigrante de Barcelona. La evolución de esta enfermedad es radicalmente diferente; mientras que en la población autóctona presenta una tendencia a la baja, coincidiendo con el descenso de nuevos de casos de SIDA, en la población inmigrante, la tendencia es al alza. La estimación de nuevos casos para el años 2009 ha sido de 168 (IC 95\% 109227) y en la población inmigrante de de 227 (IC 95\%, 180275).

Palabras clave: Incidencia de tuberculosis, previsión, regresión segmentada.

\section{ABSTRACT \\ Tuberculosis in Barcelona: \\ A Predictive model Based on Temporal Series}

Tuberculosis (TB) continues to cause millions of new cases and deaths worldwide every year. The World Health Organization (WHO) has recently estimated that in 2007 $9,273,000$ cases (incidence 139/100,000 inhabitants) and $1,772,000$ deaths were attributed to TB and, in the previous year, the absolute figure was slightly lower $(9,240,000$ cases $)$ and the incidence somewhat higher $(140 / 100,000)$, making it difficult to determine whether there was a reduction or not. The objective of this study was to apply fovecasting models to $\mathrm{TB}$, differentiating between indigenous and immigrant subjects, in a city in which the annual number of cases has been recorded since 1987.

Adjusted segmented regression (piecewise regression) was applied to the series of new cases in the indigenous and immigrant populations of Barcelona. The evolution of TB differed radically; whereas in the indigenous population there was a downward trend, coinciding with the reduction in new of cases of AIDS, in immigrants there was an upward trend. The estimated number of new cases in 2009 was 168 (95\% CI 109 $227)$ in indigenous subjects and 227 (95\% CI, $180-275)$ in immigrants.

Key words: Tuberculosis incidence, forecast, piecewise regression.

\footnotetext{
Correspondencia:

M Pilar Muñoz

Departamento de Estadística e Investigación Operativa

Universitat Politècnica de Catalunya

pilar.munyoz@upc.edu.

Telf. 934017030
} 


\section{INTRODUCCIÓN}

La tuberculosis (TB) a escala mundial sigue ocasionando cada año millones de casos nuevos y de muertes. La Organización Mundial de la Salud (OMS) ha estimado recientemente que en el 2007 se registraron 9.273.000 casos (incidencia de 139/100.000 habitantes) y 1.772 .000 muertes atribuibles a esta vieja enfermedad, y en el año precedente la cifra absoluta fue ligeramente inferior (9.240.000 casos) mientras que la incidencia fue algo superior $(140 / 100.000)^{1}$, por lo se hace difícil precisar si hay declive o no.

En Barcelona ha sido posible mantener un Programa de Prevención y Control de la Tuberculosis desde 1987. Se basa en un sistema de vigilancia epidemiológica activa que permite identificar la practica totalidad de los casos, lo que implica un seguimiento del cumplimiento del tratamiento y del estudio de contactos por parte de enfermeras de salud pública ${ }^{2}$.

El cambio demográfico experimentado en los últimos años ha atenuado el declive que se venía observando previamente. En 1991 se registró una incidencia máxima de 67,1/100.000 que fue disminuyendo progresivamente hasta el 2000 $(36,9 / 100.000)$ y en el 2007 la incidencia aún era de 31,1/100.000 y prácticamente la mitad de los casos correspondían a inmigrantes ${ }^{4}$.

La incidencia en el período estudiado ha disminuido aproximadamente el 50\%.

Los modelos predictivos basados en series temporales ya se han utilizado en Cataluña en la gripe $^{5}$ y en la enfermedad meningocócica 6 . El objetivo de este trabajo es aplicar estos modelos ${ }^{7}$ a la TB, diferenciando entre población autóctona e inmigrante, en una ciudad en la que ha sido posible determinar el número anual de casos desde 1987.

\section{MATERIAL Y MÉTODOS}

Se ha trabajado con datos diarios recogidos el Registro de casos en TB del programa de control de Barcelona, desde el 1/1/1987 hasta el 31/12/2008. Los casos registrados, en el período de estudio, son 15.270. Los casos de TB se han asignado a partir de la fecha de inicio de tratamiento y en caso de no saberse, por la fecha de declaración. Se ha agregado la incidencia cada cuatro semanas, formando así dos series temporales, una para autóctonos y otra para inmigrantes con una longitud cada una de ellas de 287 observaciones. (Figuras 1 y 2).

Dadas las características estadísticas de las series por cuatrisemanas tanto de los autóctonos como de los inmigrantes, en primer lugar se ha realizado un análisis exploratorio de la misma. A continuación se ha procedido a realizar un análisis de cambios estructurales siguiendo el procedimiento descrito en Zeilis et al (2003) ${ }^{8}$, que permite detectar los puntos de rotura que indican un cambio en la tendencia. Finalmente se ha ajustado un modelo de tendencia segmentada también denominada regresión discontinua a tramos (piecewise regression o segmented regression). La regresión lineal discontinua a tramos fue introducida originalmente por Thistlethwaite y Campbell $(1960)^{9}$ y reciente Keliber y Zeileis (2008) ${ }^{10}$ han publicado un volumen bajo un enfoque aplicado. La idea intuitiva de esta metodología es que nos permite detectar los diferentes cambios que han ocurrido en la tendencia de las declaraciones de la enfermedad a lo largo del tiempo del estudio. Esta tendencia estimada es la que nos va a permitir realizar las previsiones y su intervalo de confianza para las dos series temporales mencionadas previamente.

El análisis estadístico se ha realizado utilizando el paquete estadístico $\mathrm{R}$ de libre distribución versión 2.8.1 (http://cran.r- 
project.org). Los cambios estructurales se han detectado a partir de la librería de $\mathrm{R}$ strucchange, utilizando la función breakpoints y el análisis de tendencia mediante la función $l m$ implementada en el paquete básico stats de R.

\section{RESULTADOS}

Incidencia de TB en la población de autóctonos. En la serie de incidencia de TB en la población de los autóctonos (figura 1) se observa que no presenta una media constante a lo largo de los años del estudio ni mucho menos se puede afirmar una variabilidad constante. Hay una tendencia crecien- te hasta el año 1991 y a partir de esta fecha empieza a decrecer progresivamente la incidencia de TB en los autóctonos hasta la actualidad, presentando el descenso más brusco entre los años 1996 y 2000. Además la variabilidad también decrece, manteniéndose aproximadamente constante a partir del año 2001. La incidencia máxima de TB se produjo en la sexta cuatrisemana del año 1991 declarándose 105 casos en esa cuatrisemana. La incidencia mínima de TB en los autóctonos ha sido de 9 casos en los años 2007, cuatrisemana 1 y el año 2008, cuatrisemana 4.

Puntos de cambio de tendencia en la población autóctona. La tendencia pre-

Figura 1

Evolución temporal de las declaraciones de incidencia en la población de autóctonos (cuatrisemanas). En rojo las tendencias estimadas

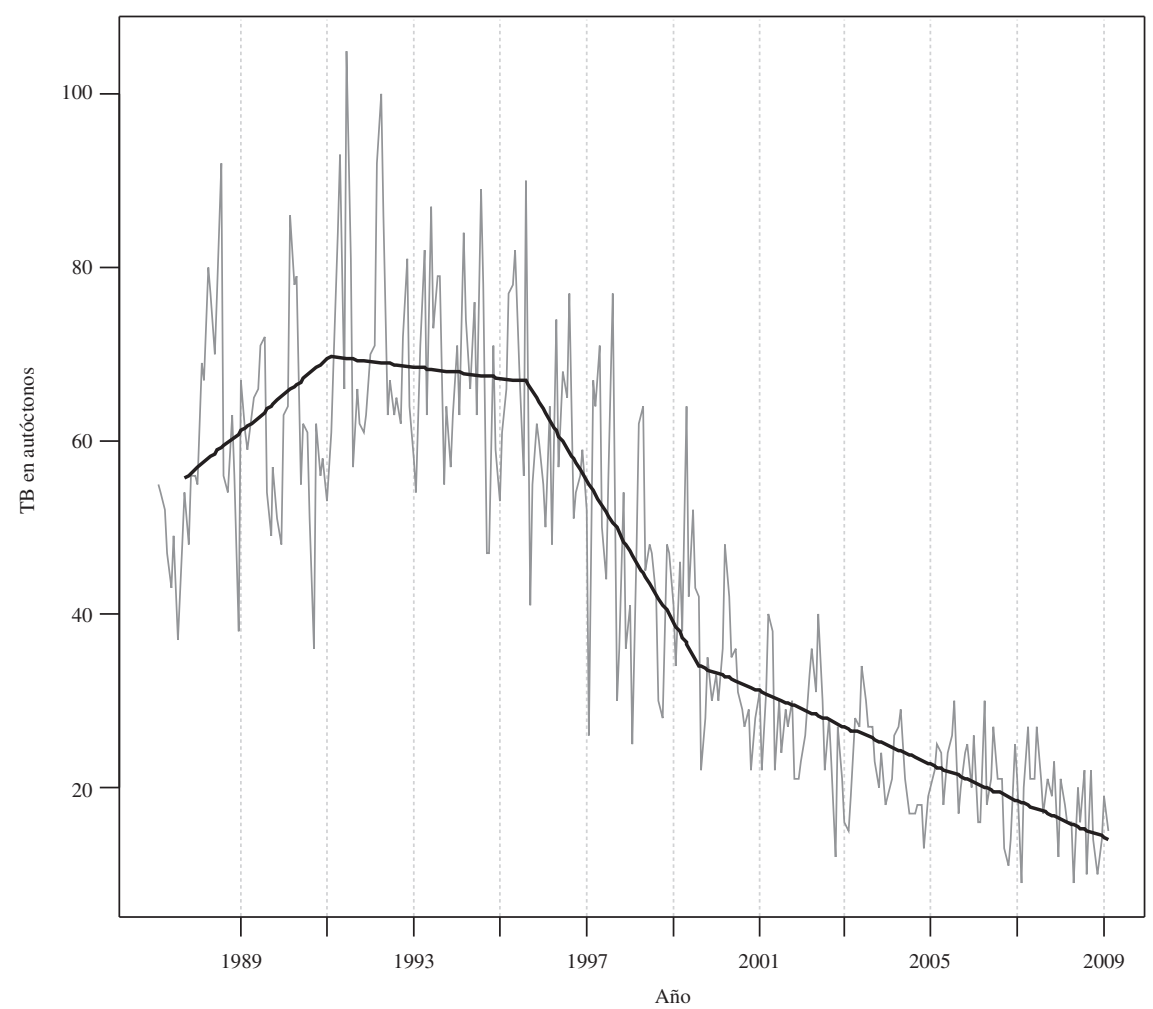




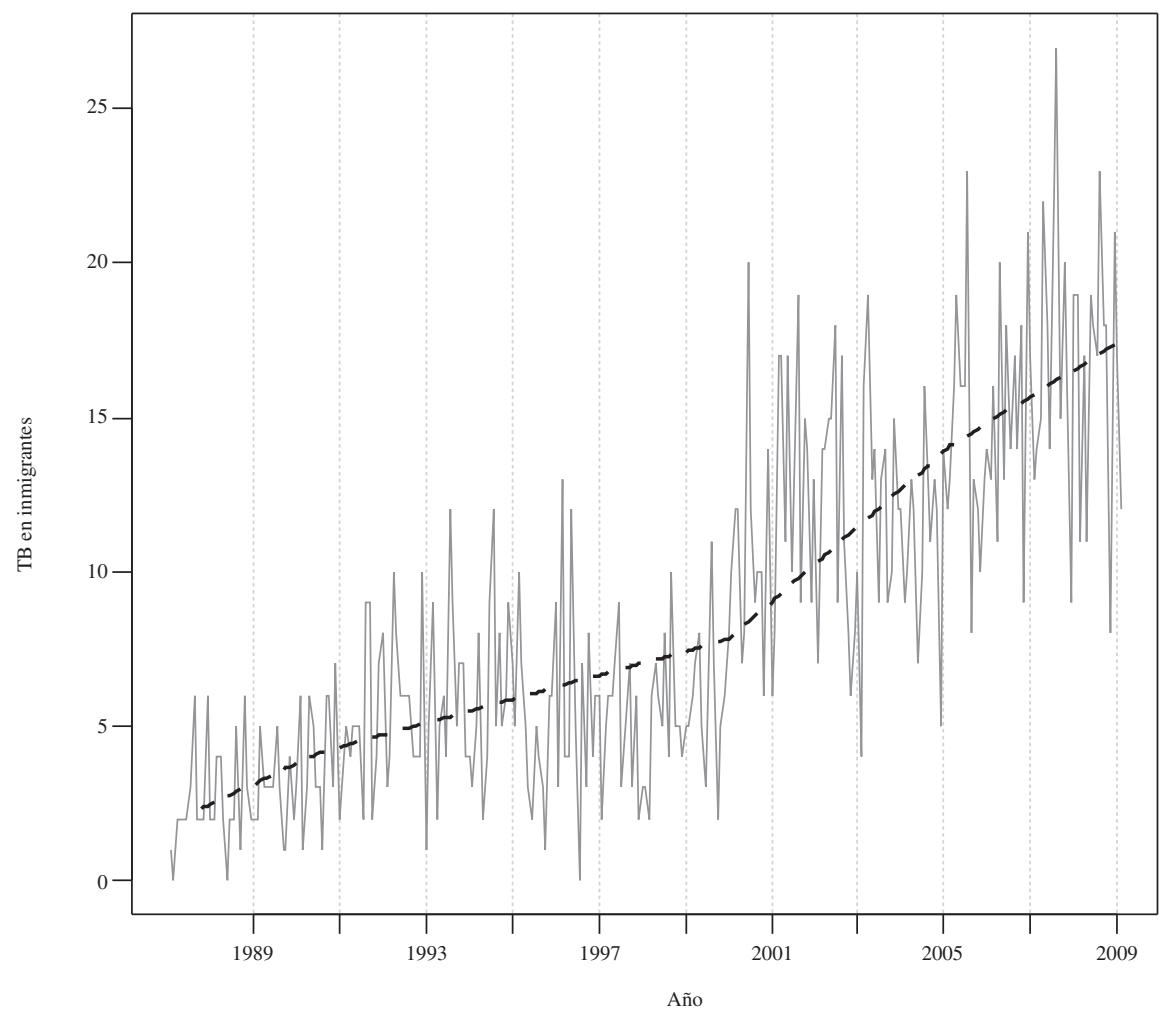

senta diferentes cambios en la de serie de nuevos casos detectados en la población autóctona, produciéndose estos puntos de rotura de la tendencia en los meses de enero de 1991 y los meses de agosto de 1995 y 1999 respectivamente. Fijémonos que hay una tendencia creciente en las declaraciones de TB hasta enero de 1991, a partir de esa fecha se mantienen ligeramente estables con un pequeño descenso hasta agosto de 1995, un pronunciado descenso de nuevos casos de TB hasta agosto de 1999 y este descenso en nuevas declaraciones continua hasta la actualidad aunque no es tan pronunciado como en el periodo 1995-1999.
En este punto es importante destacar que tanto la mortalidad por SIDA como el número de casos clarados de SIDA presentan unas tendencias similares a las comentadas de TB en la población autóctona. Observando la figura 3 se deduce que mortalidad y casos de SIDA descienden a partir del año 1995, manteniéndose ambos estables a partir del año 1999, comportamiento muy similar al que hemos detectado para la evolución de la TB en este punto.

Previsión año 2009 de nuevos casos de TB, población autóctona. A partir del análisis de tendencias realizado en el 
Figura 3

Evolución anual de los casos y del número de muertos de SIDA

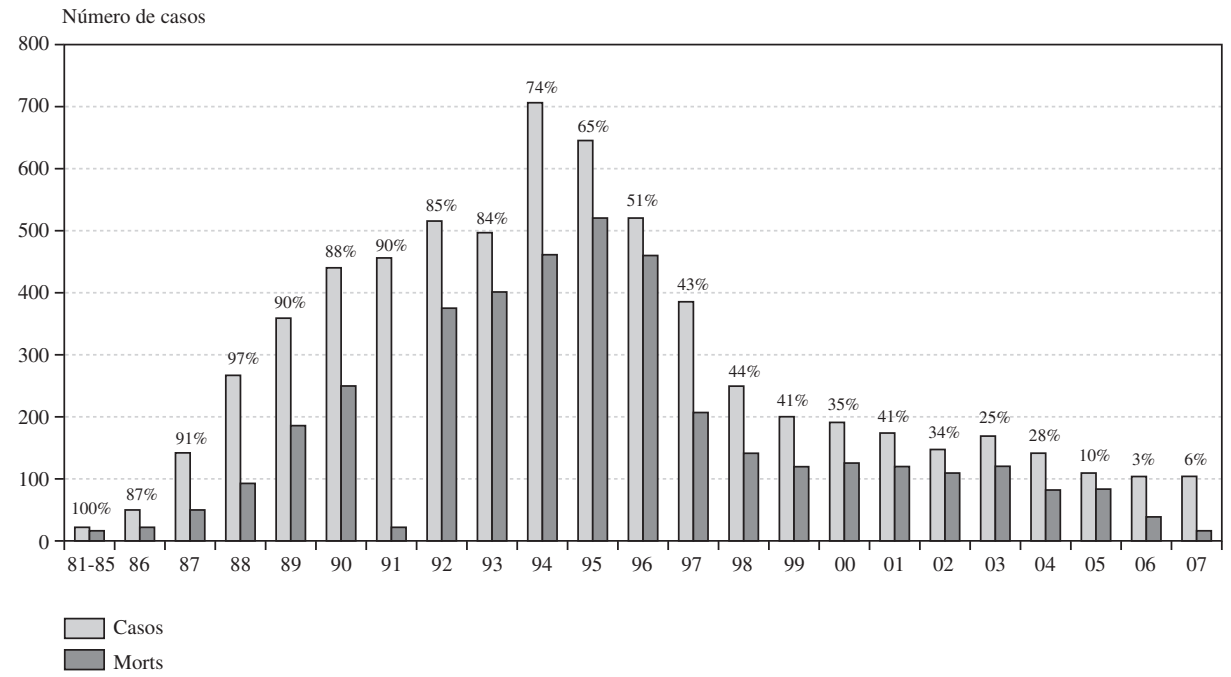

Residentes en Barcelona (1981-2007), según datos a 15/01/2008.

Fuente: Agencia de Salud Pública. Sida a Barcelona num 77.

apartado anterior se ha podido estimar que el número total de casos para el año 2009 en este colectivo será de 168 (IC 95\% 109-227).

Incidencia de TB en la población inmigrante. La incidencia de TB en la población de inmigrantes de Barcelona (figura 2) presenta una tendencia creciente a lo largo de todo el estudio y el incremento de variabilidad también es creciente. En este caso las mayores incidencias de TB se presentan a partir del año 2000, siendo la mayor incidencia en la cuatrisemana 8 del año 2006, declarándose 27 casos en esa cuatrisemana. Las incidencias mínimas en la población de inmigrantes han sido las declaradas al inicio del estudio.

Puntos de cambio de tendencia en la población de inmigrantes. Los punto de cambio de tendencia estimados para la serie de los inmigrantes se corresponden con
Agosto de 1990, Diciembre de 1999 y Febrero de 2005. En todos ellos se presenta un incremento cada vez mayor de nuevos casos declarados, aunque el más espectacular es el que se produce a partir de Diciembre de 1999.

Comparando esta serie con la del número de inmigrantes que se empadronan anualmente en la ciudad de Barcelona (Figura 4) se puede observar que la tendencia sigue al alza en ambas series y que el gran aumento en los casos de TB en esta población coincide con el incremento de este colectivo.

Previsión año 2009 de nuevos casos de TB, población inmigrante. En este punto, la estimación de las tendencias realizadas en este punto nos ha permitido predecir el número de nuevos casos en la población de inmigrantes para el año 2009 será de 227 (IC 95\%, 180-275). 
Figura 4

Evolución del número de inmigrantes empadronados anualmente en la ciudad de Barcelona (1991-2007).

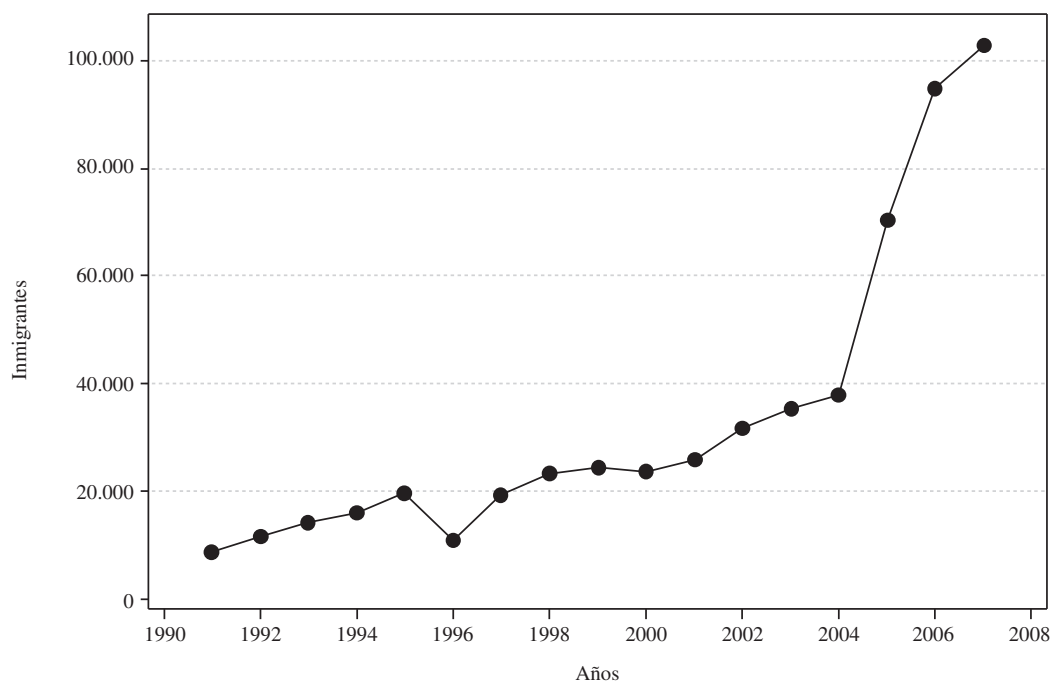

Elaboración propia a partir de los datos de inmigración del Ayuntamiento de Barcelona.

\section{DISCUSIÓN}

La conclusión más importante a destacar es que las tendencias de nuevos casos de TB presentan patrones diferentes en la población de autóctonos y la de inmigrantes. Mientras que en la primera hay un descenso significativo de nuevos casos a partir del año 1995, coincidiendo con el descenso en la morbilidad y mortalidad por SIDA en Barcelona, la segunda presenta un incremento continuo de nuevos casos de TB en la población de inmigrantes que en este caso coincide con el incremento de inmigración en esta ciudad.

A pesar de que la incidencia ha disminuido alrededor del $50 \%$, el declive anual ha sido menor a partir del año 2000. Es previsible que en los próximos años el porcentaje de inmigrantes con TB supere el 50\% del total de casos detectados, patrón típico observado desde hace años en ciudades y países del mundo desarrollado.
La estimación de los nuevos casos de TB en ambas poblaciones se ha realizado mediante la regresión segmentada o discontinua.

Este procedimiento es sencillo de implementar y muy útil para predecir futuros valores de series temporales con diferentes tendencias. La interpretación de los resultados es muy fácil. Ha sido utilizado con éxito en temas biosanitarios, entre otros autores por Chen y Mynett (2004) ${ }^{11}$ y recientemente por Garabrant et al. (2009) ${ }^{12}$.

\section{BIBLIOGRAFÍA}

1. WHO Report 2009. Global tuberculosis control epidemiology, strategy, financing. WHO/HTM/ TB/2009.411. Disponible en: http://www.who.int/tb/ publications/global_report/2009/en/index.html

2. WHO Report 2009. Global tuberculosis control epidemiology, strategy, financing. WHO/HTM/ TB/2009.411. Disponible en: http://www.who.int/tb/ publications/global_report/2009/en/index.html 
3. Rodrigo T, Caylà JA, Galdós-Tangüis H, García de Olalla P, Brugal MT, Jansà JM. Proposing indicators for evaluation of tuberculosis control programmes in large cities based on the experience of Barcelona. Int J Tuberc Lung Dis. 2001; 5: 432-40.

4. Orcau A, Rius C, García de Olalla P, Caylà JA. La Tuberculosi a Barcelona. Informe 2007. Barcelna: Publicaciones de la Agència de Salut Pública, 2008. Disponible en: http://www.aspb.es/quefem/docs/Tuberculosi_2007.pdf

5. Domínguez A, Muñoz P, Martínez A, Orcau A. Monitoring mortality as an indicator of influenza in Catalonia, Spain. J Epidemiol Community Health. 1996 Jun; 50(3): 293-8.

6. Domínguez A, Muñoz P, Cardeñosa N, Martínez A, Caylà J; Meningococcal Disease Study Group. Time-series analysis of meningococcal disease in Catalonia. Ann Epidemiol. 2007;17: 654-62.

7. Muñoz M.P. Previsión de la evolución de un paciente. Med Clin (Barc) 2004;122(Supl 1):45-50.
8. Zeileis A., Kleiber C., Krämer W., Hornik K. Testing and Dating of Structural Changes in Practice, CSDA, 2003, 44:109-123.

9. Thistlethwaite, D., Campbell D. Regression-discontinuity analysis: An alternative to the ex post factor experiment. Journal of Educational Psychology 1960, 51: 309-317.

10. Kleiber, C., Zeileis, A. Applied Econometrics with R. 2008. Springer.

11. Chen Q., Mynett A.E., Predicting Phoeocystic geobosa bloom in Dutch costal waters by decision trees and nolinear piecewise regression. Ecological Modelling, 2004, 176: 277-290.

12. Garabiant DH., Aylward L.L., Berent S., Chen Q., Timchalle C., Burns C.J., Hays S.M., Albers J.W. Cholinesterase inhibition in chlorpunrifos workers: characterization of biomarkers of exposure and responde in relation to urinary TCPy. Journal of Exposure Science \& Enviornmental Epidemiology. 2009, 19: 634-642. 
